\title{
Cutaneous Force Feedback as a Sensory Subtraction Technique in Haptics
}

\author{
Domenico Prattichizzo, Member, IEEE, Claudio Pacchierotti, Student Member, IEEE, and Giulio Rosati
}

\begin{abstract}
A novel sensory substitution technique is presented. Kinesthetic and cutaneous force feedback are substituted by cutaneous feedback (CF) only, provided by two wearable devices able to apply forces to the index finger and the thumb, while holding a handle during a teleoperation task. The force pattern, fed back to the user while using the cutaneous devices, is similar, in terms of intensity and area of application, to the cutaneous force pattern applied to the finger pad while interacting with a haptic device providing both cutaneous and kinesthetic force feedback. The pattern generated using the cutaneous devices can be thought as a subtraction between the complete haptic feedback (HF) and the kinesthetic part of it. For this reason, we refer to this approach as sensory subtraction instead of sensory substitution. A needle insertion scenario is considered to validate the approach. The haptic device is connected to a virtual environment simulating a needle insertion task. Experiments show that the perception of inserting a needle using the cutaneous-only force feedback is nearly indistinguishable from the one felt by the user while using both cutaneous and kinesthetic feedback. As most of the sensory substitution approaches, the proposed sensory subtraction technique also has the advantage of not suffering from stability issues of teleoperation systems due, for instance, to communication delays. Moreover, experiments show that the sensory subtraction technique outperforms sensory substitution with more conventional visual feedback (VF).
\end{abstract}

Index Terms - Sensory substitution, cutaneous force feedback, wearable devices, haptic devices, needle insertion , tactile force feedback

\section{INTRODUCTION}

A novel approach to sensory substitution in haptics is presented. Sensory substitution is used in teleoperation to display forces using other modalities such as audio or visual feedback (VF) or other forms of haptic feedback (HF) such as vibrotactile feedback. Sensory substitution techniques are frequently used in medical applications [1]. In this paper we focus on a simulated environment for teleoperated needle insertion in soft tissues.

In recent years studies on needle insertion in soft tissues have attracted considerable attention due to their promising applications in minimally invasive percutaneous procedures such as biopsies [2], blood sampling [3], neurosurgery [4] [5], and brachytherapy [6] [7]. The effectiveness of a treatment depends on the accuracy of percutaneous insertion [8] [7], especially when working on critical areas like the brain.

Force feedback is an important navigation tool during surgical needle advancement. It allows to detect local mechanical properties of the tissue being penetrated and distinguish between expected and abnormal resistance due, for example, to the unexpected presence of vessels [9]. An interesting study on the effect of teleoperation on perception abilities of human operators on the stiffness of the tissue has been recently presented in [10].

In bilateral teleoperation, stability and transparency can be significantly affected by communication latency of the teleoperation loop which dramatically reduces the

- D. Prattichizzo and C. Pacchierotti are with the Department of Information Engineering, University of Siena, Via Roma 56, Siena, Italy and with the Department of Advanced Robotics, Istituto Italiano di Tecnologia, Via Morego 30, Genova, Italy. G. Rosati is with the Department of Management and Engineering, University of Padua, Padua, Italy.
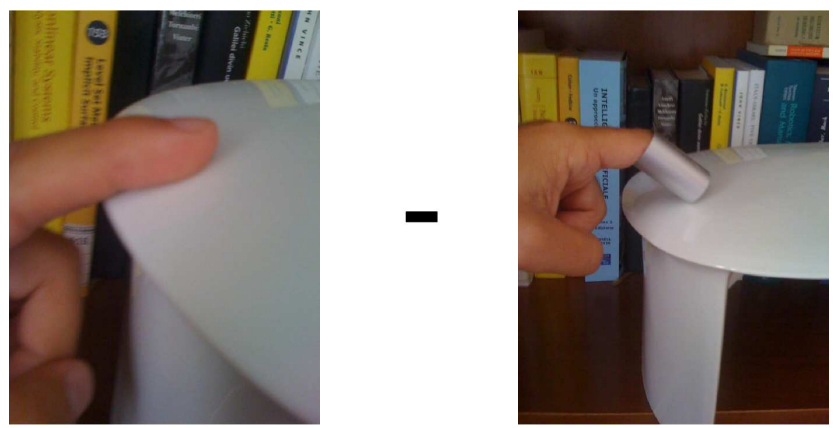

Fig. 1: While touching an object, the human feels both kinesthetic and cutaneous stimuli (left); isolating the fingertip skin with a thimble makes the user perceive kinesthetic interaction mainly (right). In the interaction with haptic devices, the subtraction of kinesthetic feedback from the mixed stimuli brings to cutaneous only feedback.

effectiveness of haptic feedback in case of stiff remote environments [11] [12]. This limitation can be alleviated requiring passivity of the interconnected system [13], using wave variable transformation [14] [15] or designing proper control systems [16] [17]. However, designing proper control algorithms to guarantee stability cannot be considered as an intrinsically safe approach. To prevent serious mechanical faults such as actuator failures on the master side, which can generate undesired and unsafe motions of the slave robot, different approaches must be considered. In particular, we need to consider techniques dealing more with the hardware design than the control architecture of the teleoperation loop.

In the literature, a possible hardware design approach 
consists in using passive components such as brakes [18] or passive isometric input devices [19]. However, passive input devices have rendering limitations and may lead to large steady-state errors in teleoperation tasks. To reduce the effects of these limitations, researchers implemented energy-bounding algorithms [20] or used motors and brakes together with the aim of obtaining a safer teleoperation while preserving system transparency [21].

Another interesting approach consists in avoiding to use any actuator for force feedback on the master side and alternatively providing the force feedback using sensory substitution techniques. Force feedback is not kinesthetic anymore and the haptic loop becomes intrinsically stable since no force is fed back to the operator through the haptic device. Sensory substitution techniques replaces this lack of kinesthetic feedback with other forms of feedback such as vibrotactile [22] [23], auditory, and/or visual feedback [24] [25].

The sensory subtraction technique presented in this work can be casted in a sensory substitution framework but there are relevant differences which are worth underlining to motivate the use of the term subtraction. The main idea is that, instead of rendering forces with a complete haptic feedback, consisting of cutaneous and kinesthetic components, we present to the human operator the cutaneous component only, without the kinesthetic part. A novel wearable cutaneous force feedback device has been developed for this aim. Differently from other works on cutaneous feedback (CF), the device presented in this paper is not of the array type as discussed, for instance, in [26] and [27], but it allows to apply vertical stresses to the finger pad, similarly to the gravity grabber presented in [28]. The role of cutaneous feedback in haptics, compared to kinesthetic feedback, has been recently discussed and exploited, for example, in [29], where Wijntjes et al. discussed the effects of kinesthetic and cutaneous information for curvature discrimination, in [30], where Ferber et al. investigated cutaneous and kinesthetic cues to maintain exercise intensity on a stair climber machine and in [31] where the problem of missed kinesthetic feedback in wearable haptics is discussed. All these papers underline how relevant is the cutaneous feedback when compared to kinesthesia.

In this work, we will show how the proposed cutaneous-feedback sensory subtraction technique, other than being intrinsically stable, improves the teleoperation performances with respect to other sensory substitution techniques such as the one using visual feedback. Preliminary results on the sensory subtraction approach were presented for an industrial application in [32], where the the gravity grabber presented in [28] was used. In this paper, we discuss the results of experiments on sensory subtraction for needle insertion in which we use new wearable cutaneous devices.

The rest of the paper is organized as follows: the idea of sensory subtraction is discussed in Sec. 2 along with the description of the cutaneous device. The teleoperated needle insertion application is introduced in Sec. 3. Experiments carried out to validate the proposed sensory subtraction technique are presented and discussed in Sec. 4. Finally, Sec. 5 addresses concluding remarks and perspectives of the work.

\section{Sensory subtraction}

The idea behind sensory subtraction originates from the observation that the stimuli received by the user while holding a haptic handle consists of a cutaneous and a kinesthetic component. Cutaneous sensation is produced by pressure receptors in the skin and they are useful to recognize the local properties of objects such as shape, edges, embossings and recessed features, thanks to a direct measure of the intensity and direction of the contact forces [33]. On the other hand, kinesthesia provides the user with information about the relative position of neighboring parts of the body, by means of sensory organs in the muscles and joints [34].

In this work, we propose to use an interface able to generate cutaneous force feedback only instead of the complete haptic feedback (both kinesthetic and cutaneous), during the execution of a simple robot-assisted surgical task. In particular, we will substitute the haptic force feedback with its cutaneous component provided by a device able to apply normal forces to the finger pad. With respect to traditional haptic feedback, we expect this simple form of feedback to make the teleoperation stable, and to allow the operator to perform the motion task in an equally intuitive way, as the cutaneous force feedback is perceived where it is expected and provides the operator with a direct and colocated perception of the contact force even if it is only cutaneous and not kinesthetic.

Of course, when we use the cutaneous force feedback devices, the kinesthetic sensation is still present because the hand and the arm move but not because a kinesthetic force feedback device is acting on the user. In other words, using a cutaneous force feedback device, we want to remove the kinesthetic feedback produced by the actuators of the haptic device, rather than eliminating the kinesthetic interaction.

We expect the proposed feedback modality to yield better results, in terms of task performance, with respect to other forms of sensory substitution. For this reason, the novel feedback modality will be compared not only to haptic feedback, but also to a common sensory substitution technique, in which force feedback is substituted by a visual representation of the contact force.

We could refer to our approach as sensory substitution because the mixed kinesthetic-cutaneous feedback usually provided by a haptic device is here substituted with part of the cutaneous feedback. However, it is worth underlining that here the stimulation fed back to the user is similar, in terms of intensity and area of application, to the one perceived while interacting with an actuated handle. This approach is different from other 


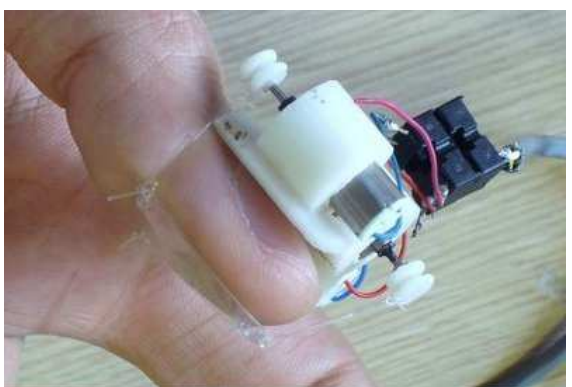

Fig. 2: The wearable cutaneous device used to apply forces normal to the operator's finger pad.

sensory substitution techniques in which the area and/or the type of stimuli are different from the ones being replaced.

By considering that the handle of a haptic device would provide both kinesthetic and cutaneous force feedback, and that the area where the force is applied is equivalent (i.e., the finger pad), using our approach the user receives a subset of the typical stimuli provided by a haptic device. This is why we refer to the proposed approach as sensory subtraction (see Fig. 1).

\subsection{The wearable fingertip cutaneous force feed- back device}

The prototype of the cutaneous force feedback device used in our experiments is shown in Fig. 2. It consists of two main parts: the first one is on the dorsal side of the finger and supports three small electrical motors; the other has a contact patch with the volar skin surface of the fingertip. The two parts are connected by three cables. The motors, by controlling the lengths of the cables, are able to press the patch on the user's fingertip. As a result, a force is generated simulating the contact of the fingertip with the surface of an object or a handle, as in Fig. 3. The direction and amount of the force reflected to the user is changed by properly controlling cable lengths [35].

This device applies forces between the volar skin surface and the nail. In contrast, when humans actively exert fingertip forces during manipulation of real objects, forces operate essentially between the phalangeal

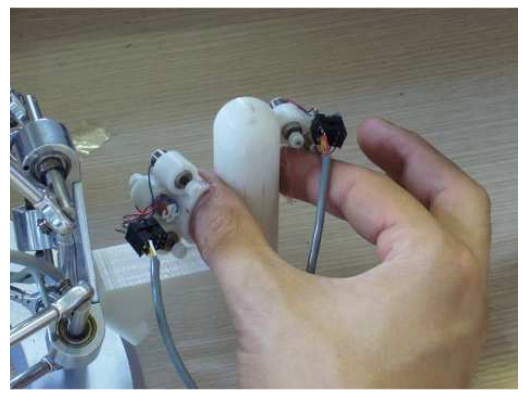

Fig. 3: The haptic handle grabbed by the operator using two fingers and two wearable cutaneous devices.

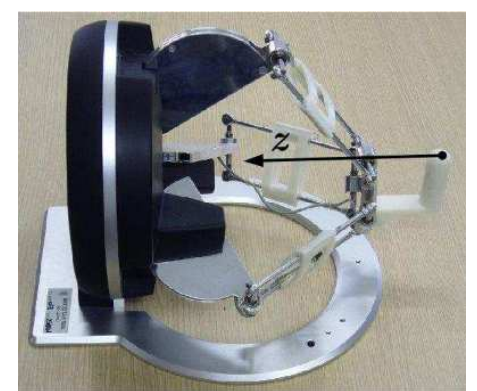

Fig. 4: The haptic device Omega 3, with three clamps limiting the motion of the handle along the $z$-axis only.

bone and the volar skin surface. Birznieks et al. [33] demonstrated that the deformational changes in the fingertip are similar under the two conditions, i.e., when stimulated by a device similar to the one proposed in this work, the fingertip will deform as if the subject was actively applying forces against a real object. For this reason, the cutaneous stimulation produced by the wearable device can be considered to some extent equivalent to that perceived while actively interacting with a haptic handle (Fig. 3).

The device described above belongs to the category of wearable haptic devices and it is an evolution of the first idea presented by K. Minamizawa et al. [28]. In particular, the evolution consists of using three motors instead of two, and a 3-dof parallel manipulator architecture [36] to render forces at the finger pad. For the purpose of this work, the wearable device in Fig. 2 was controlled as a 1-dof system (all motors pulled the cables together), so that only forces in the sagittal plane of the finger were actuated, roughly normal to the longitudinal axis of the distal phalanx.

\subsection{Sensory subtraction - a demonstrator}

In our experiments, we used four prototypes of the fingertip cutaneous device and a commercial haptic device. The operator wears two cutaneous devices on one hand, one on the thumb and one on the index finger, and grabs the handle as shown in Fig. 3. Two additional cutaneous devices are worn on the thumb and index finger of the contralateral hand. The haptic device is the Omega 3 by Force Dimension, to which three clamps were applied to reduce the degrees of freedom from three to one (the $z$ axis in Fig. (4). Also, a plastic handle was attached to its end-effector to allow the operator to grab the device with two fingers (Fig. 3).

During the experiments, the hardware was operated in two different modalities. The first one is referred to as complete haptic feedback, where the feedback force is provided by the Omega 3 while the wearable devices are switched off. In this way, by interacting with the handle, the operator receives mixed kinesthetic and cutaneous stimuli, i.e., the complete haptic feedback.

The second modality is referred to as cutaneous-only feedback, where the proposed sensory subtraction tech- 
nique is implemented. In this modality, the Omega 3 is used only to track the motion of the hand with its encoders and does not apply any active force to the operator (the actuators of the Omega 3 are switched off). At the same time, the wearable devices are used to reproduce the cutaneous sensation associated to the manipulation task being simulated. For instance, a feedback force directed towards the negative direction of the $z$-axis (see Fig. 4) is substituted by applying a normal stress to the index finger. Conversely, a force directed towards the positive direction of the $z$-axis is substituted by a normal stress applied to the thumb. To investigate the role of feedback localization with respect to the hand involved in the task, either the devices on the active hand or those worn on the contralateral hand are alternatively activated.

\section{A MEDICAL APPLICATION OF SENSORY SUB- TRACTION}

In this work, we test the sensory subtraction approach on a simulated scenario of needle insertion in a soft tissue. Force feedback is helpful during needle advancement to detect local mechanical properties of the tissue and to distinguish between expected and abnormal resistance due, for example, to the unexpected presence of vessels, or to the action of active constraints, that are usually introduced to protect areas of the soft tissue that must be avoided to prevent damage of tissue and of its functionality. This is the case, for instance, of brain surgery, in which tissue manipulation in special areas can cause serious injury to patients.

Active constraints, commonly referred to as virtual fixtures [37], are software functions used in assistive robotic systems to regulate the motion of surgical implements. The motion of the surgical implement, the needle in our case, is still controlled by the surgeon, but the system constantly monitors its motion and takes some actions if the surgical tool fails to follow a predetermined procedure. Virtual fixtures play two main roles: they can either guide the motion or strictly forbid the surgeon from reaching certain regions [38]. A guiding virtual fixture attenuates the motion of the surgical implement in some predefined directions to encourage the surgeon to conform to the procedure plan. A forbidden-region virtual fixture is a software constraint that seeks preventing the needle from entering a specific region of the workspace. In this paper, we consider an example of virtual fixtures protecting forbidden regions. This is a common scenario for biopsies, deep brain stimulation and functional neurosurgery.

When performing keyhole neurosurgery the needle can be steered using a haptic device such as the Omega 3, and the motion of the needle will be along one direction only [9], [39]. The device used in the experiments is reported in Fig. 3 A special handle is attached to the end-effector and the motion is constrained to one degree of freedom, by means of three clamps attached to the

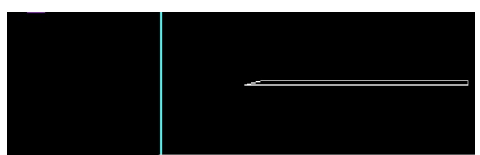

(a) no needle-tissue contact

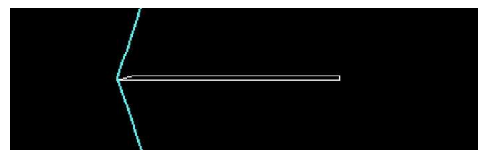

(b) needle reaches the tissue

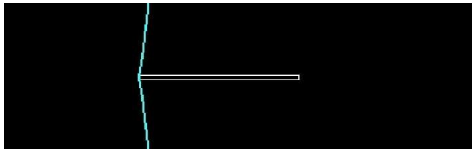

(c) needle penetrates the tissue

Fig. 5: Screenshots of the virtual environment, composed by the needle (white), driven by the operator, and the deformable tissue (light blue). The portion of the needle already inserted in the tissue is not shown on screen (c).

parallel structure of the device. The Omega 3 is typically used as a haptic device of the impedance type: the position of the needle, moved by the human operator, is measured, and a force signal is fed back to the user through the actuation system. The force feedback accounts for either the remote contact interaction of the slave robot, in a classical teleoperation scenario, or by the virtual environment, in case of simulations.

In the proposed setup, the haptic handle teleoperates the needle in a virtual environment simulating the insertion in a soft tissue with virtual fixtures. The needle moves along a single axis (the $z$-axis of the haptic device) as in Fig. 5. where the needle and the surface of the tissue are shown. The contact force between the needle and the tissue is calculated according to the visco-elastic model presented in Sec. 3.1

\subsection{Soft tissue modeling and haptic rendering}

The operator remotely steering the needle feels a resistive force, while penetrating the tissue, due to its viscoelastic properties, and an opposite force while trying to pull the needle out. In real scenarios, these forces are either measured from force sensors or estimated from other parameters.

In this work a simple simulation of the soft tissue is used. The aim of this work is not to design an accurate tissue simulator based for instance on FEM techniques [40] but to validate the proposed sensory subtraction approach.

A spring $K_{t}=2 \mathrm{~N} / \mathrm{m}$ and a damper $B_{t}=5 \mathrm{Ns} / \mathrm{m}$ are used to model the contact force $F_{t}$ between the needle and the tissue, while a spring $K_{v f}=3000 \mathrm{~N} / \mathrm{m}$ is used to model the contact force $F_{v f}$ between the needle and the virtual fixture. For the sake of simplicity, we assume that the mass of the tissue $M_{t}=1 \mathrm{~kg}$ is concentrated 
at the contact point. The viscous coefficient of the body beneath the tissue is $V_{t}=0.7 \mathrm{Ns} / \mathrm{m}$.

As for the haptic rendering, the interaction is designed according to the god-object model [41] and the position of the Omega handle is linked to the needle position $z_{n}$ moving in the virtual environment. The initial position of the surface of the tissue is set to $\bar{z}_{t}=20 \mathrm{~mm}$ and the virtual fixture is located at $\bar{z}_{v f}=123 \mathrm{~mm}$.

Tissue position $z_{t}$ changes according to the interaction with the needle, which is able to penetrate the surface only when the haptic force $F_{h}$ is larger than a predetermined threshold $\left(F_{p}=0.1 \mathrm{~N}\right)$. To have a wider workspace, a scale factor of 3 between the position of the needle in the virtual environment and the operator's hand is used.

It is possible to discriminate four different operating conditions for the needle-tissue interaction model here presented:

- no contact (see Fig. 5a),

- contact without penetration (see Fig. 5b),

- penetration within the safe area (see Fig. $5 \mathrm{c}$ ), and

- penetration and contact with the virtual fixture

In the first case, since the needle is out of the tissue, the model is designed to feed back no force to the operator and the surface of the tissue tends to return to its predetermined initial position $\bar{z}_{t}$. The dynamics of the interaction for the no contact case is

$$
\left\{\begin{array}{l}
M_{t} \ddot{z}_{t}=-K_{t}\left(z_{t}-\bar{z}_{t}\right)-B_{t} \dot{z}_{t} \\
F_{h}=0 .
\end{array}\right.
$$

When the needle touches the tissue, but the force $F_{h}$ is not yet sufficient to penetrate it, the tissue surface is deformed by the movement of the needle. In this case, the dynamic model and the contact force to be fed back to the operator are

$$
\left\{\begin{array}{l}
z_{t}=z_{n}, \\
F_{h}=-K_{t}\left(z_{t}-\bar{z}_{t}\right)-B_{t} \dot{z}_{t} .
\end{array}\right.
$$

As soon as $F_{h}>F_{p}$, the needle penetrates the surface and while the needle is inside the tissue, the dynamics and the contact force are computed as

$$
\left\{\begin{array}{l}
M_{t} \ddot{z}_{t}=-K_{t}\left(z_{t}-\bar{z}_{t}\right)-B_{t} \dot{z}_{t}-V_{t}\left(\dot{z}_{t}-\dot{z}_{n}\right), \\
F_{h}=-V_{t}\left(\dot{z}_{t}-\dot{z}_{n}\right) .
\end{array}\right.
$$

If the operator steers the needle towards the unsafe workspace area delimited by the virtual fixture, a force will be fed back to the operator in order to avoid the penetration of the needle in the forbidden area:

$$
F_{v f}=-K_{v f}\left(z_{n}-\bar{z}_{v f}\right) .
$$

Note that the virtual fixture generates a force feedback which is more than $10^{3}$ times larger than the force felt while in contact with the soft tissue.

The haptic device measures the position of the operator's hand (with a resolution of $0.01 \mathrm{~mm}$ ), sends it to the controller and then the virtual environment computes the force feedback and the dynamics of the tissue. The controller then sends the force back to the user through either the haptic device or the substitutive (cutaneous or visual) modality.

\subsection{Design of experiments}

Four alternative feedback modalities were compared in the experiments: (complete) haptic feedback, applied by the actuators of the haptic handle, visual feedback in substitution of haptic feedback, cutaneous-only feedback in substitution of haptic feedback, applied by the wearable devices either on the fingers holding the handle or on the fingers of the contralateral hand. The visual feedback consisted in showing a horizontal bar representing the contact force registered at the needlepoint.

The subjects were asked to wear the four cutaneous devices for the whole duration of the experiments, and to grasp the handle with their right hand as shown in Fig. 3. The subject's hand was positioned with its longitudinal axis at 90 degrees from the Omega $z$-axis. The position of the subject's hand with respect to the joystick was checked before the beginning of each experiment. To prevent changes in the perceived direction of the feedback force generated by the Omega 3, the subjects were instructed to move the forearm rather than the wrist while moving the device. During the experiments, the subjects maintained the initial orientation of the fingers with respect to the handle, which was the only natural way of grasping the handle for the 1-dof task 1 .

The task consisted in inserting the needle into the soft tissue and stopping the motion when a virtual fixture was perceived. After $5 \mathrm{~s}$ of continuous contact with the fixture, the system played a sound beep. The subjects were instructed to pull the needle out of the tissue as soon as the sound was heard. In all the considered tasks, regardless of particular feedback modality employed, visual feedback on needle insertion was provided to the subjects, showing the part of the needle out of the tissue and the surface of the tissue: the virtual fixture and the portion of the needle inside the tissue were not visible (see Fig. 5).

No information on the feedback modalities was provided, neither on their nature (except from visual feedback in substitution of force feedback) nor on the particular order with which they were going to be presented to the subject. Both the sequence of the feedback modalities and the position of the virtual fixture were randomized.

Three different experiments were implemented:

- experiment \#1: twenty-four repetitions of the needle insertion task described above;

1. A modification of the way the fingers grasp the handle would imply that the perceived direction of the feedback force changes if haptic feedback is used, whereas it would not change with cutaneousonly feedback. This issue must be considered while trying to extend the sensory subtraction paradigm to multi-dof tasks, since the results may be affected by this change of direction of the force vector. Thus, the position of the operator's hand with respect to the input device must be carefully monitored before and during the experiments. 


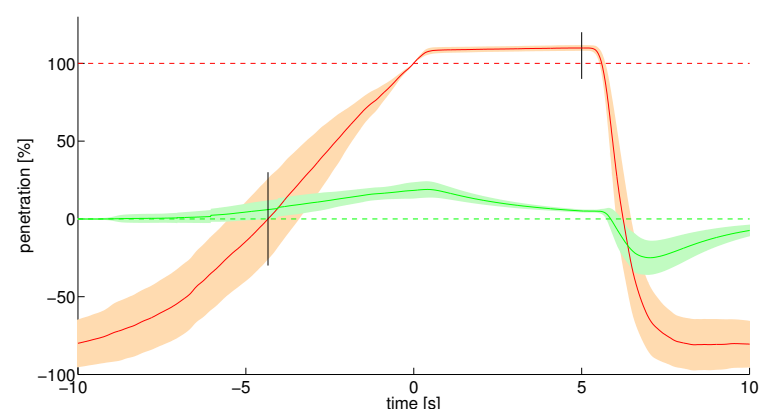

(a) Visual feedback (VF)

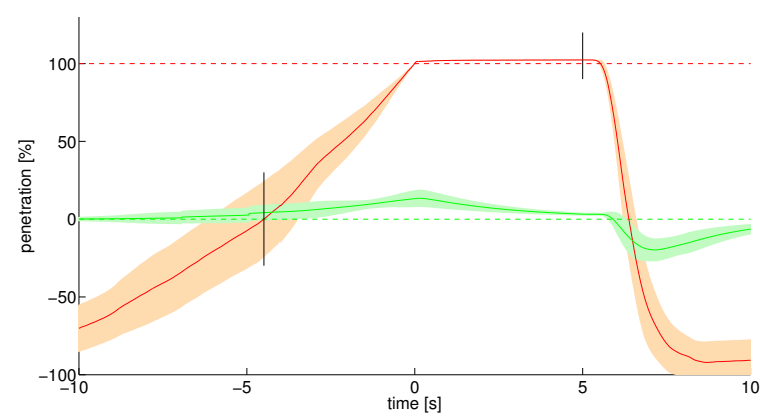

(b) Haptic feedback (HF)

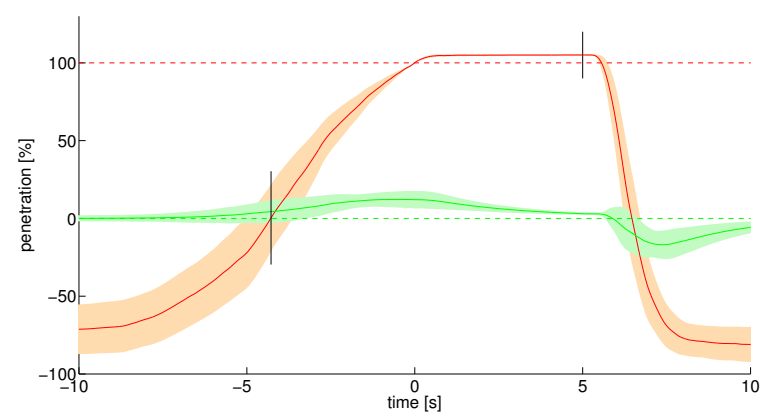

(c) Cutaneous feedback on the hand holding the handle (CF)

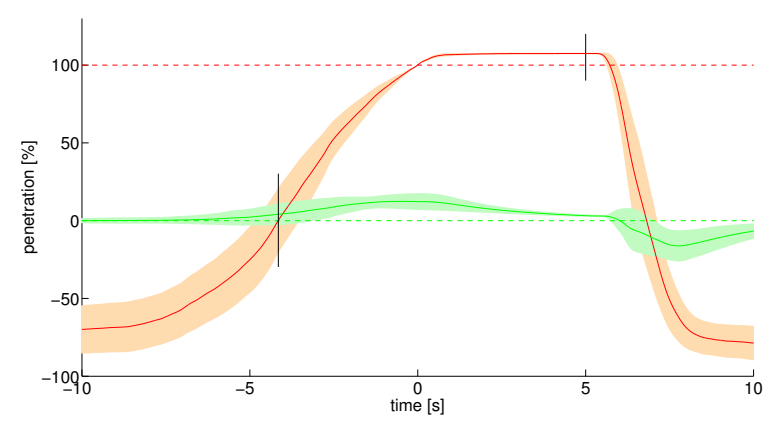

(d) Cutaneous feedback on the contralateral hand (CCF)

Fig. 6: Penetration of the needle (red patch) and position of tissue surface (green patch) versus time for experiment \#1. Average trajectories among subjects and their standard deviations are plotted. The position of the virtual fixture (dashed red line) and the initial position of tissue surface (dashed green line) are shown as well. The black lines represent the instants when the average trajectory enters the tissue (left line) and when the sound beep is played (right line).
- experiment \#2: two additional repetitions of the needle insertion task, during which the position of the virtual fixture was changed suddenly and unexpectedly;

- experiment \#3: same as experiment \#1, but in presence of a time delay in the haptic loop.

The first experiment aimed at demonstrating that, on the one hand, there is no relevant degradation of performance in the haptic interaction task (i.e., inserting the needle) when a normal force is fed back to the user's fingertip holding the handle, using the cutaneous devices in substitution of the feedback generated by a haptic device. On the other hand, this experiment aimed at demonstrating that using the cutaneous devices can lead to better performances with respect to other forms of sensory substitution, such as visual feedback in substitution of force feedback, in which the alternative feedback modality is different in nature from the one being substituted. Moreover, the experiment investigated if the fact that cutaneous force feedback provides a reliable form of feedback is due only to presenting a force to the user, or also to the fact that the feedback information is applied to the fingertips which are responsible for handling the needle during the experiments.

The second experiment aimed at showing that using the cutaneous devices prevents the handle (and so the needle) from moving in unwanted directions in case of sudden and unpredictable changes of the position of the virtual fixture.

The third experiment aimed at confirming the well known result that there are no instability behaviours, not even in presence of delays, while using cutaneous force feedback devices.

\section{EXPERIMENTAL RESULTS}

\subsection{Experiment $\# 1$ : comparison of the feedback modalities}

Sixteen participants (13 males, 3 females, age range $21-$ 28 ) took part in the experiment, all of whom were righthanded. Eight of them had previous experience with haptic interfaces. None of the participants reported any deficiencies in the perception abilities (including vision, hearing, touch and proprioception). Each participant made 24 repetitions of the needle insertion task, with six randomized trials for each feedback mode:

- visual feedback by the horizontal bar (task VF);

- haptic feedback (kinesthetic and cutaneous) by the haptic device (task HF);

- cutaneous feedback by the wearable devices, applied to the hand holding the handle (task CF);

- cutaneous feedback by the wearable devices, applied to the contralateral hand (task CCF).

The experiment lasted 9.13 minutes on average, including the two additional trials for experiment $\# 2$, which followed the twelfth and the 24th repetitions of experiment \#1 (see Sec.4.2 for details). A total of 26 tasks were 


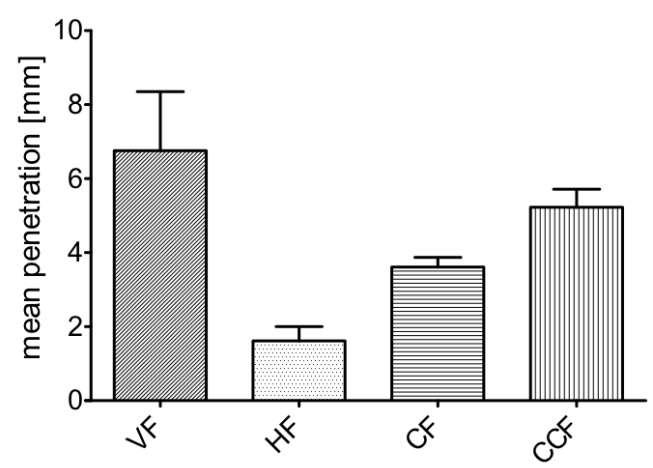

Fig. 7: Experiment \#1: average penetration beyond the virtual fixture (mean and SD), for the visual (VF), haptic (HF) and cutaneous feedback modes (CF, CCF). A null value of this metric indicates high accuracy in reaching the target depth.

performed by each subject, 24 of which were included in the results of experiment \#1.

With the aim of comparing the different feedback modalities, the position $z_{n}$ of the needle, steered by the operator's hand, was recorded and the penetration into the virtual fixture $p=\bar{z}_{v f}-z_{n}$ was calculated. The average penetration $\bar{p}$ and the maximum penetration $\bar{p}_{M}$ were analyzed 2 . Such values provide a measure of accuracy (average penetration) and of overshoot (maximum penetration) in reaching the target depth. A null value in both metrics denotes the best performance, while a positive value indicates that the subject overrun the target. Both measures can be considered particularly relevant to the surgical task, as an excessive penetration of the needle can result in permanent damage of tissues.

Fig. 6 shows the positions of the needle (red patch) and of the tissue surface (green patch) versus time. The time bases of different trials were synchronized at the time the needle first enters the fixture $(t=0)$, while positions were divided by the depth of the virtual fixture, which varied randomly among trials, and are presented as percentage. Trajectories were averaged among subjects for each feedback modality, and average trajectories plus/minus standard deviations are shown. The position of the virtual fixture (dashed red line, 100 percent) and the initial position of tissue surface (dashed green line, 0 percent) are shown as well. The black lines represent the instants when the average trajectory enters the tissue (left line) and when the sound beep is played (right line).

Fig. 7 and Fig. 8 show the average and maximum penetrations beyond the fixture for each feedback modality (means and standard deviations are plotted). All column data passed the D'Agostino-Pearson omnibus K2 normality test. Comparison of the means among the feedback modalities was tested using one-way, repeated measures analysis of variance (ANOVA). The

2. Data resulting from different trials of the same task, performed by the same subject, were averaged before comparison with other tasks.

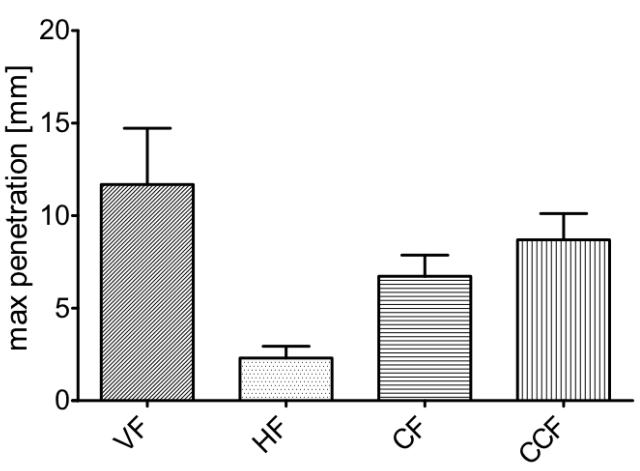

Fig. 8: Experiment \#1: maximum penetration beyond the virtual fixture (mean and SD), for the visual (VF), haptic (HF) and cutaneous feedback modes (CF, CCF). A null value of this metric indicates no overshoot in reaching the target depth.

means of average penetration (Fig. 7. $F_{3,45}=106.5$, $P<0.0001)$ and the means of maximum penetration (Fig. 8, $F_{3,45}=81.89, P<0.0001$ ) differed significantly among the feedback modalities. Posthoc analyses (Bonferroni's multiple comparison test) revealed statistically significant difference between all pairs of columns, both in terms of average penetration (Fig. 7, $P<0.001$ for all pairs) and in terms of maximum penetration of the needle (Fig. 8, $P<0.05$ for CF vs CCF, and $P<0.001$ for all other pairs). Results indicate that the proposed sensory subtraction modality (CF) yields an intermediate performance between haptic feedback (HF, best performance) and visual feedback (VF, worst case), in terms of both average and maximum penetration beyond the virtual fixture. These results demonstrate also that the cutaneous devices provide a more reliable form of feedback if applied to the fingertips which are responsible for holding the end-effector (CF) with respect to contralateral hand stimulation (CCF), suggesting that the localization of cutaneous feedback is crucial in this setting. Nonetheless, cutaneous feedback is more efficacious than visual feedback (VF) even when it is applied to the contralateral hand (CCF), indicating that not only the localization but also the nature of the sensation provided is relevant to task performance.

Fig. 9 shows the average time elapsed between the instant the needle penetrates the tissue and the instant it reaches $5 \mathrm{~s}$ of continuous contact with the virtual fixture. Column data failed to pass the normality test, so the Friedman non-parametric test was used to analyze variance. Results indicate that there is no statistically significant difference between the feedback modalities in this metric $(P>0.1)$. We may read this result by saying that the subjects became equally confident with all the feedback modalities proposed.

\subsection{Experiment \#2: dynamic virtual fixture}

This experiment evaluated the effect on needle position of a sudden and unpredictable change of the position of 


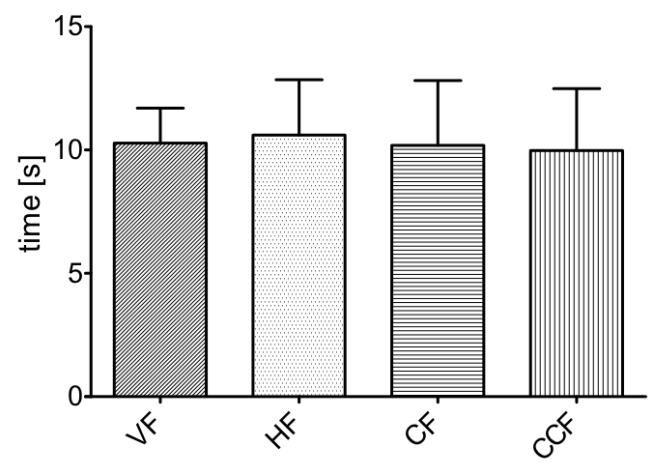

Fig. 9: Experiment \#1: time elapsed (mean and SD) between the first contact with the tissue and the sound played after $5 \mathrm{~s}$ of continuous contact with the virtual fixture, for the visual (VF), haptic (HF) and cutaneous feedback modes (CF, CCF).

the virtual fixture, in the presence of the four feedback modalities described before (visual, haptic and the two cutaneous). In this new test, the needle insertion task was the same as that described in Sec. 4.1. However, after $5 \mathrm{~s}$ of continuous contact, the depth of the virtual fixture was increased unexpectedly, so the virtual environment suddenly fed back no guiding force to the user. At the same time, the sound beep was produced as in the other repetitions of the needle insertion task, signaling the subject to extract the needle. The two circumstances provided conflicting information to the user. In fact, the user was initially instructed to keep contact with the fixture, so at the one hand the sudden change in the guiding force suggested to increase needle depth. On the other hand, the sound signaled to extract the needle.

The test was performed during two additional trials of experiment \#1. To ensure the surprise effect, each of the subjects who took part in the experiment \#1 performed only two additional trials (using two different feedback modalities). A total of 32 trials were recorded for experiment \#2: 8 trials per each feedback modality, performed by eight different subjects. The first additional trial was run after the 12 th trial of experiment $\# 1$, the second after the 24th. No information was provided to the subjects about the additional trials, which followed immediately the previous ones. A $30 \mathrm{~s}$ rest was given to all subjects after the first additional trial, before continuing with the second part of experiment \#1. Subjects did not know that the position of the virtual fixture was going to change and that they were performing a different task with respect to the others.

Fig. 10 shows the differences $\Delta p$ between the maximum penetration registered after the perturbation and the average penetration observed in the $5 \mathrm{~s}$ before (continuous contact). All column data passed the D'Agostino-Pearson omnibus K2 normality test. Comparison of the means among the feedback modalities was tested using one-way ANOVA (no repeated measures). The means differed significantly among the feedback

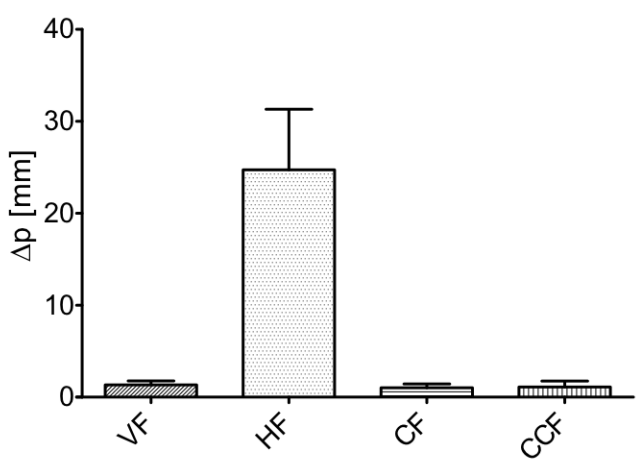

Fig. 10: Experiment \#2: difference (mean and SD) between the maximum penetration, after the movement of the virtual fixture, and the average penetration registered before (during continuous contact), for the visual (VF), haptic (HF) and cutaneous feedback modes (CF, CCF).

modalities $\left(F_{3,28}=100.3, P<0.0001\right)$. Posthoc analyses (Bonferroni's multiple comparison test) revealed statistically significant difference between haptic feedback (HF) and each alternate modality (VF, CF, CCF, $P<0.001$ ). Results indicate that the presence of kinesthetic feedback may induce significantly greater unwanted motions of the needle with respect to the three non-kinesthetic feedback modes used in the experiments (the visual and the two cutaneous-only modalities). In fact, when the fixture moves in haptic mode (HF), the subject's arm is counteracting an external force which suddenly drops.

Fig. 11 shows the positions of the needle (red patch) and of the tissue surface (green patch) versus time for all the groups of experiment \#2. Data were synchronized, normalized and averaged among subjects as for the charts of Fig. 6

\subsection{Experiment \#3: stability with time delay}

As other sensory substitution techniques, the main advantage of the proposed cutaneous-feedback sensory subtraction is that it makes the haptic loop intrinsically stable. No instability behaviors occur, even in presence of large delays.

To support this hypothesis, a new set of experiments was implemented, in which the same protocol used in the experiment described in Sec. 4.1 was used for the needle insertion task, including the types of feedback employed and number of repetitions (24) per subject, but here a delay of $50 \mathrm{~ms}$ was introduced in the haptic loop between the virtual environment and either the haptic handle, the cutaneous devices or the visual rendering of force. Recent literature denotes the relevance of delays in teleoperated surgical tasks [42]. It is worth noting that instability of haptic feedback in the presence of time delays can be fixed with a wave variable transformation [14], [15], [43]. Nonetheless, to emphasize the intrinsic stability of cutaneous feedback, this method was not used in the trials. 
Ten participants ( 8 males, 2 females, age range $20-26$ ) took part in the experiment, all of whom were righthanded and five of whom had previous experience with haptic interfaces. None of the participants reported any deficiencies in the perception abilities (as defined before). The experiment lasted 8.39 minutes on average.

Fig. 12 shows the positions of the needle (red patch) and of the tissue surface (green patch) versus time for experiment \#3. Data were synchronized, normalized and averaged among subjects as for Fig. 6. By comparing the charts with those in Fig. 6, we can notice that the instability occurred only with haptic feedback, i.e., only in the presence of kinesthetic feedback. Significant oscillations of the needle are likely to bring not only a greater penetration of the needle in the virtual fixture, but also a longer task completion time?

Fig. 13 shows the maximum penetration beyond the fixture in the presence of the delay. Haptic feedback group data (HF) failed to pass the normality test, so the Friedman non-parametric test was used to analyze variance. The test indicated statistically significant difference between the feedback modalities $(P<0.0001)$. Posthoc analyses (Dunn's multiple comparison test) revealed statistically significant difference between haptic feedback (HF) and both cutaneous modalities $(\mathrm{CF}, P<0.001$; CCF, $P<0.05)$ and between cutaneous feedback $(\mathrm{CF})$ and visual feedback (VF, $P<0.001)$. Results indicate that the subjects, while receiving the complete haptic feedback in the presence of a time delay, reached a significantly greater peak penetration in the virtual fixture with respect to that obtained while receiving feedback from the wearable cutaneous devices, regardless the localization of cutaneous feedback. The same result was obtained when the subjects received visual feedback of force instead of cutaneous feedback on the fingers which are responsible for handling the needle.

Fig. 14 shows, for each feedback modality and in the presence of the time delay, the mean time elapsed between the first penetration in the tissue and the instant the needle reaches $5 \mathrm{~s}$ of stable contact with the virtual fixture. Haptic feedback and cutaneous feedback group data failed to pass the normality test, so the Friedman non-parametric test was used to analyze variance. The test indicated statistically significant difference between the feedback modalities $(P<0.0001)$. Posthoc analyses (Dunn's multiple comparison test) revealed statistically significant difference between haptic feedback (HF) and all other feedback modalities (VF, $P<0.01$; $\mathrm{CF}, P<0.05$; CCF, $P<0.001)$. Results indicate that the time needed to accomplish the task was significantly greater while receiving the kinesthetic feedback with respect to the other non-kinesthetic feedback modalities. Such a difference had not been observed in the absence of time delays (Fig. 9), and must be related to instability.

3. A short movie of an experimental run showing the instability issue can be downloaded at http://goo.gl/9vDqC

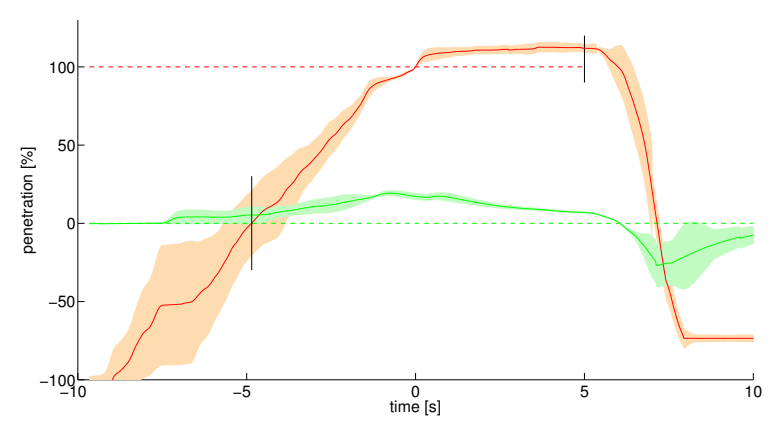

(a) Visual feedback (VF)

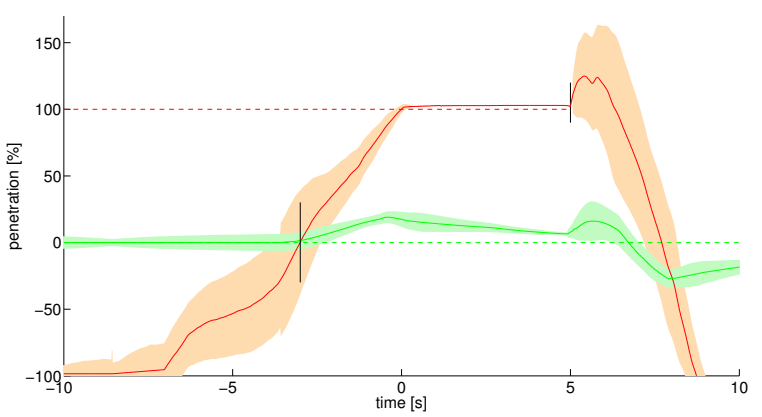

(b) Complete haptic feedback (HF)

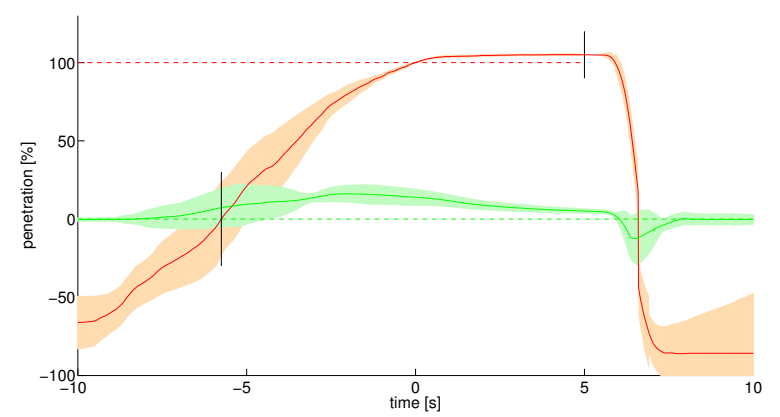

(c) Cutaneous feedback on the hand holding the handle (CF)

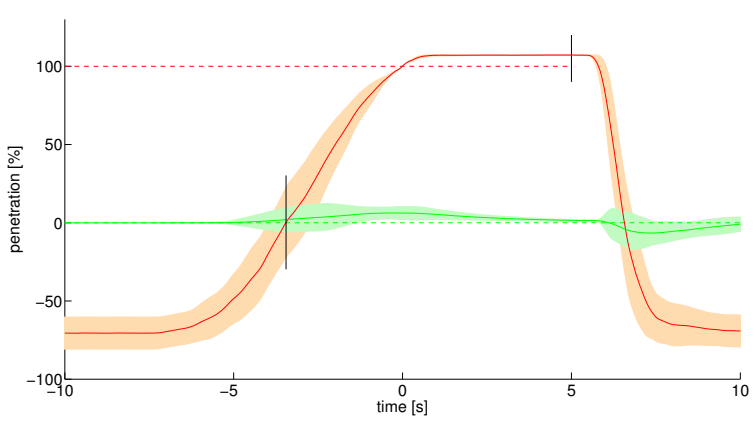

(d) Cutaneous feedback on the contralateral hand (CCF)

Fig. 11: Penetration of the needle (red patch) and position of tissue surface (green patch) versus time for experiment \#2, with the virtual fixture suddenly removed after $5 \mathrm{~s}$ of continuous contact. Average trajectories among subjects and their standard deviations are plotted. The position of the virtual fixture (dashed red line) and the initial position of tissue surface (dashed green line) are shown as well. The black lines represent the instants when the average trajectory enters the tissue (left line) and when the virtual fixture is removed and the sound beep is played (right line). 


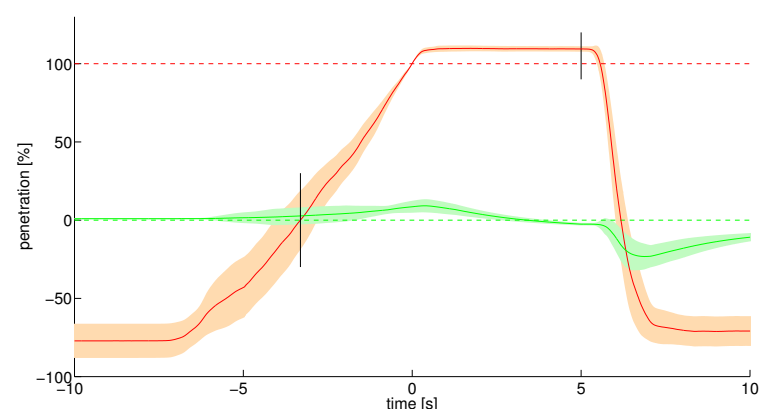

(a) Visual feedback (VF)

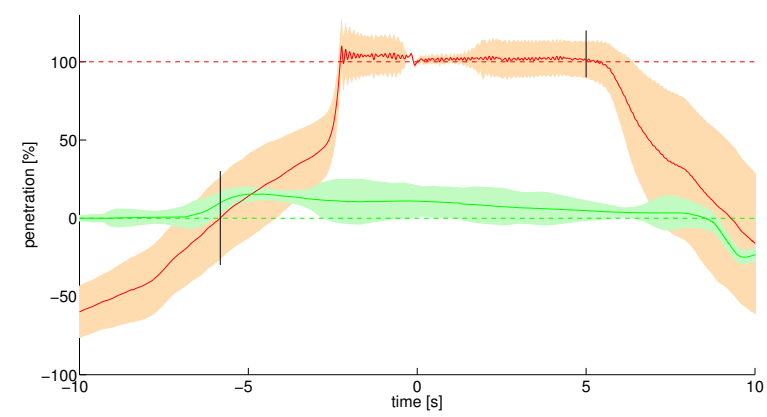

(b) Complete haptic feedback (HF)

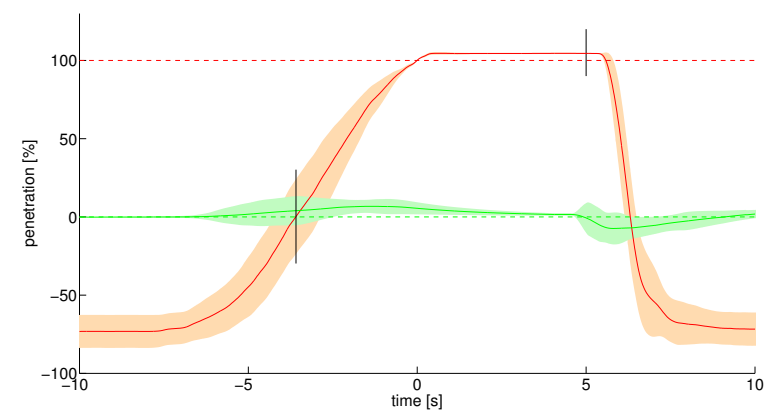

(c) Cutaneous feedback on the hand holding the handle (CF)

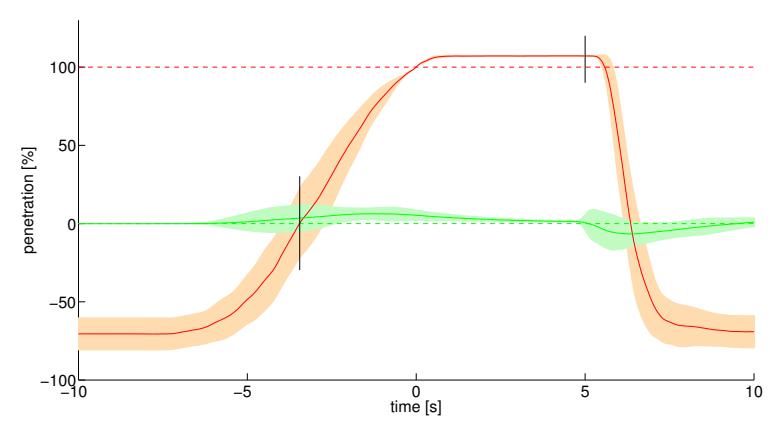

(d) Cutaneous feedback on the contralateral hand (CCF)

Fig. 12: Penetration of the needle (red patch) and position of tissue surface (green patch) versus time for experiment \#3, with a $50 \mathrm{~ms}$ network delay in the haptic loop. Average trajectories among subjects and their standard deviations are plotted. The position of the virtual fixture (dashed red line) and the initial position of tissue surface (dashed green line) are shown as well. The black lines represent the instants when the average trajectory enters the tissue (left line) and when the sound beep is played (right line).

\subsection{Discussion}

The first experiment evaluated the effectiveness of the sensory subtraction technique proposed in the paper. The results of this experiment indicate that the subjects, while receiving visual feedback (VF) in substitution of force feedback, reached a significantly greater average and maximum penetration in the virtual fixture (worst performance) in comparison with that obtained while receiving either complete haptic (HF) or cutaneousonly feedback (CF and CCF). The last two modalities provided intermediate performance between visual and haptic feedback. No difference between groups was observed in terms of task completion time.

As expected, haptic feedback outperformed all the other feedback modes. The cutaneous-only modality proved itself to be a more intuitive form of feedback than other sensory substitution techniques, regardless the localization of the cutaneous devices (either on the hand performing the task or on the contralateral hand). When the cutaneous force feedback was applied to the contralateral hand (i.e., the one not involved in controlling the motion of the input device), performance was worst in terms of penetration of the virtual fixture with respect to the case when the cutaneous force feedback was applied to the acting hand. A possible mechanistic interpretation could be that the cutaneous feedback applied to the contralateral hand needs time for transcallosal transmission to reach the hemisphere controlling the operating hand. In fact, the feedback reaches the hemisphere of the brain not involved in the motor control of the hand moving the input device, and for this reason requires more time to be transformed in motor action [44].

It is worth underlying that larger penetration into the virtual fixture corresponds to a higher force fed back by the virtual environment, applied by either the haptic device, the cutaneous actuators or displayed using the horizontal bar for sensory substitution with visual modality. Also note that the larger penetration observed when cutaneous force feedback was used may be partly due to the delay of the cutaneous actuators employed in the tests, which can be quantified in $\sim 45 \mathrm{~ms}$.

These results suggest that the novel feedback modality can be successfully used in substitution of traditional haptic feedback, with a minor decay of performance with respect to visual sensory substitution techniques. Not only the type of feedback, cutaneous rather than visual, but also the place where it is applied is important. The best performance is obtained when the cutaneous devices are worn on the hand involved in the task, i.e., when they provide the user with a subset of the stimuli produced by the input device in complete haptic mode. This result can be explained by considering that the area of application of the force and the particular design of the cutaneous devices proposed provide the user with a direct and intuitive measure of the contact force being substituted, thus producing a more natural interaction 


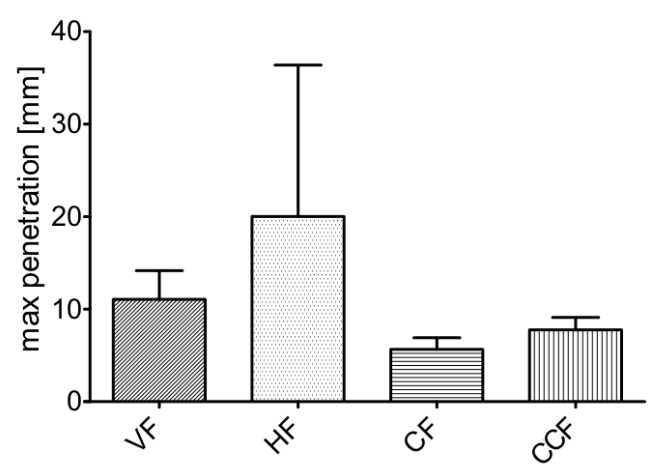

Fig. 13: Experiment \#3: maximum penetration beyond the virtual fixture (mean and SD), for the the visual (VF), haptic (HF) and cutaneous feedback modes (CF, CCF), with a $50 \mathrm{~ms}$ network delay in the loop.

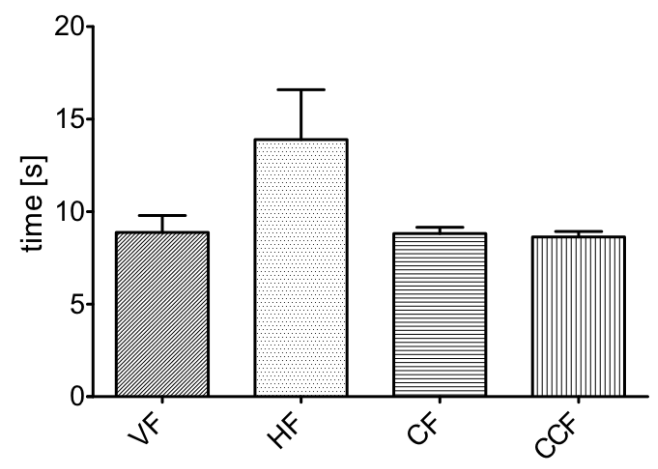

Fig. 14: Experiment \#3: time elapsed between the first contact with the tissue and the sound beep, for the visual (VF), haptic (HF) and cutaneous feedback modes (CF, $\mathrm{CCF})$, with a 50 ms network delay in the haptic loop.

with the device.

One interesting result observed with sensory subtraction is that, during the first experiment, performance degraded when kinesthetic information was removed. One possible explanation is that the external force subtracted played a role in arm dynamics during the execution of the needle insertion task. In particular, the virtual force helped the subject in stopping hand motion when the virtual fixture was reached, which is the main reason for using virtual fixtures indeed. Conversely, in the proposed touch-only modality, no physical aid is provided to the user to accomplish the task, so arm motion derives entirely from motor control. The resulting benefit is that unwanted motions can be drastically reduced in critical situations.

On the other hand, without adequate sense of touch, achieving normal and top performance in tasks that require high levels of dexterity is extremely difficult, if not impossible [45]. Moreover, even simple touch information can be effective both in virtual and in real environments. For example, major gains in body posture control in real environments can be obtained from min- imal touch information applied to a fingertip [46]. This may explain why touch-only tasks were better executed than the substituted visual tasks.

One major advantage of sensory subtraction is that, despite the fact that the interaction is closer to haptic rendering, no unwanted movements are likely to be produced during the execution of guided tasks. This achievement, that is corroborated by the results of experiment $\# 2$, is particularly crucial in critical applications such as robot-aided surgery, in which unwanted movements of the surgeon's hand induced by force feedback may produce serious damages to the patient. The absence of unwanted movements, even in the case of sudden and unpredictable changes of the position of the virtual fixture, can be explained by considering that kinesthetic feedback was completely eliminated in the cutaneous feedback modality, so the user could maintain a stable contact with the virtual fixture without exerting an active force on the handle.

The last experiment showed that, in the presence of a transmission delay, complete haptic feedback can bring the haptic loop near to instability, as significant oscillations of needle position occurred, whereas cutaneous (and visual) feedback allows a stable contact with the virtual fixture surface. The occurrence of instability with a relatively small time delay may be due to the particular setting of the experimental device used in the experiments. However, the fact that kinesthesia can bring instability in haptic teleoperation in the presence of time delays is well-know in the literature on haptics as discussed in the introductory section.

Another drawback of using complete haptic feedback in presence of transmission delays is the longer time needed to complete the task. Statistical analysis on task completion times showed that, in case of no delay, there are no significant differences between the four different feedback modalities, while in the presence of a network delay, task completion time using haptic feedback can be significantly greater than that obtained using cutaneousonly feedback.

\section{CONCLUSION AND FUTURE WORK}

We showed that cutaneous force feedback applied to the thumb and index finger pads during the manipulation of a handle in teleoperation tasks can be effectively used to substitute complete cutaneous and kinesthetic feedback in haptics. The main advantage of using cutaneous force feedback displays is that the stability of the haptic loop is intrinsically guaranteed. This can be very convenient for critical applications like robotic surgery. Note also that actuation for cutaneous displays usually requires less power and it is less bulky than that required to provide haptic feedback, with a direct effect on simplifying mechanical design and reducing costs.

The main drawback of the proposed approach is that, like for other sensory substitution techniques, the realism of the interaction is weaker when compared to complete 
haptic feedback but, differently from other substitution techniques, the proposed one has the advantage of being perceived exactly where it is expected and provides the operator with a direct and co-located perception of the contact force even if it is only cutaneous and not kinesthetic. This is a possible explanation of the better performances of the proposed sensory subtraction technique.

Although the mechanical design leads to simple light and portable cutaneous devices, work is in progress to improve their level of wearability thus reducing the impact of using such devices. Work is in progress to design new cutaneous displays with better dynamic performances, in order to design and conduct additional psychophysical experiments to assess other relevant parameters, like for instance the just noticeable difference (JND) for mechanical properties [47]. Another important aspect of future research is to evaluate the possibility of presenting to the user not only cutaneous cues but also the kinesthetic feedback with a scaled intensity. Setting the scaling factor will be an interesting aspect of this research. Also the combination of the cutaneous-only paradigm with other modalities, like auditory feedback, is worthy being investigated. Finally, while in this study we did not consider the possibility of applying any vibratory signal to the cutaneous display since this was not compatible with the sensory subtraction idea, work is in progress to compare the cutaneous force feedback, as driven by the sensory subtraction technique, to other sensory substitution techniques using vibrotactile signals.

\section{ACKNOWLEDGMENTS}

This work was partly supported by the European Commission with the Collaborative Project grant FP7- ICT2009-6-270460 "ACTIVE: Active Constraints Technologies for Ill- defined or Volatile Environments". The authors are grateful to Rudy Manganelli and Francesco Chinello from University of Siena for their help in realizing the experiments.

\section{REFERENCES}

[1] A. Okamura, "Haptic feedback in robot-assisted minimally invasive surgery," Current opinion in urology, vol. 19, no. 1, p. 102, 2009.

[2] J. Bishoff, D. Stoianovici, B. Lee, J. Bauer, R. Taylor, L. Whitcomb, J. Cadeddu, D. Chan, and L. Kavoussi, "Rcm-paky: Clinical application of a new robotic system for precise needle placement," Journal of Endourology, vol. 12, p. S82, 1998.

[3] A. Zivanovic and B. Davies, "A robotic system for blood sampling," IEEE Transactions on Information Technology in Biomedicine, vol. 4 , no. 1 , pp. 8-14, 2000.

[4] K. Masamune, E. Kobayashi, Y. Masutani, M. Suzuki, T. Dohi, H. Iseki, and K. Takakura, "Development of an mri-compatible needle insertion manipulator for stereotactic neurosurgery," Computer Aided Surgery, vol. 1, no. 4, pp. 242-248, 1995.

[5] P. Rizun, P. McBeth, D. Louw, and G. Sutherland, "Robot-assisted neurosurgery," Surgical Innovation, vol. 11, no. 2, p. 99, 2004.

[6] S. DiMaio and S. Salcudean, "Needle insertion modeling and simulation," IEEE Transactions on Robotics and Automation, vol. 19, no. 5, pp. 864-875, 2003.
[7] J. Hing, A. Brooks, and J. Desai, "Reality-based needle insertion simulation for haptic feedback in prostate brachytherapy," in Proc. IEEE International Conference on Robotics and Automation. ICRA., vol. 1, 2006, pp. 619-624.

[8] N. Abolhassani, R. Patel, and M. Moallem, "Needle insertion into soft tissue: A survey," Medical engineering $\mathcal{E}$ physics, vol. 29, no. 4, pp. $413-431,2007$.

[9] D. Lorenzo, E. Momi, I. Dyagilev, R. Manganelli, A. Formaglio, D. Prattichizzo, M. Shoham, and G. Ferrigno, "Force feedback in a piezoelectric linear actuator for neurosurgery," The International Journal of Medical Robotics and Computer Assisted Surgery, vol. 7, no. 3, pp. 268-275, 2011.

[10] I. Nisky, A. Pressman, C. Pugh, F. Mussa-Ivaldi, and A. Karniel, "Perception and action in teleoperated needle insertion," IEEE Transactions on Haptics, vol. 4, no. 3, pp. 155-166, 2011.

[11] D. Lawrence, "Stability and transparency in bilateral teleoperation," IEEE Transactions on Robotics and Automation, vol. 9, no. 5, pp. 624-637, 1993.

[12] K. Hashtrudi-Zaad and S. Salcudean, "Transparency in timedelayed systems and the effect of local force feedback for transparent teleoperation," IEEE Transactions on Robotics and Automation, vol. 18, no. 1, pp. 108-114, 2002.

[13] D. Lee and M. Spong, "Passive bilateral teleoperation with constant time delay," IEEE Transactions on Robotics, vol. 22, no. 2, pp. 269-281, 2006

[14] G. Niemeyer and J. Slotine, "Stable adaptive teleoperation," IEEE Journal of Oceanic Engineering, vol. 16, no. 1, pp. 152-162, 1991.

[15] Y. Ye and P. Liu, "Improving haptic feedback fidelity in wavevariable-based teleoperation orientated to telemedical applications," IEEE Transactions on Instrumentation and Measurement, vol. 58, no. 8, pp. 2847-2855, 2009.

[16] R. Anderson and M. Spong, "Bilateral control of teleoperators with time delay," IEEE Transactions on Automatic Control, vol. 34, no. 5, pp. 494-501, 1989.

[17] R. Adams, M. Moreyra, and B. Hannaford, "Stability and performance of haptic displays: Theory and experiments," in Proc. ASME International Mechanical Engineering Congress and Exhibition 1998, vol. 1, 1998, pp. 227-234.

[18] B. Black and W. Book, "Dynamic compensating controller for passive haptic manipulators in teleoperation," in Proc. IEEE International Conference on Robotics and Automation, vol. 1, 2009, pp. $1485-1491$.

[19] A. Lecuyer, S. Coquillart, A. Kheddar, P. Richard, and P. Coiffet, "Pseudo-haptic feedback: Can isometric input devices simulate force feedback?" in Proc. IEEE Virtual Reality, vol. 1, 2000, pp. 83-90.

[20] J. Kim and J. Ryu, "Robustly stable haptic interaction control using an energy-bounding algorithm," The International Journal of Robotics Research, vol. 29, no. 6, p. 666, 2010.

[21] F. Conti and O. Khatib, "A new actuation approach for haptic interface design," The International Journal of Robotics Research, vol. 28, no. 6, p. 834, 2009.

[22] R. Schoonmaker and C. Cao, "Vibrotactile force feedback system for minimally invasive surgical procedures," in Proc. IEEE International Conference on Systems, Man and Cybernetics, vol. 3, 2006, pp. 2464-2469.

[23] M. Massimino and T. Sheridan, "Sensory substitution for force feedback in teleoperation," Presence: Teleoperators and Virtual Environments, vol. 2, no. 4, pp. 344-352, 1993.

[24] M. Tavakoli, R. Patel, and M. Moallem, "Haptic feedback and sensory substitution during telemanipulated suturing," in Proc. of Eurohaptics and Symposium on Haptic Interfaces for Virtual Environment and Teleoperator Systems (World Haptics), vol. 1, 2005, pp. 543-544.

[25] M. Kitagawa, D. Dokko, A. Okamura, and D. Yuh, "Effect of sensory substitution on suture-manipulation forces for robotic surgical systems," Journal of Thoracic and Cardiovascular Surgery, vol. 129, no. 1, pp. 151-158, 2005.

[26] C. Wagner, S. Lederman, and R. Howe, "A tactile shape display using rc servomotors," in Proc. 10th Symposium on Haptic Interfaces for Virtual Environment and Teleoperator Systems, vol. 1, 2002, pp. $354-355$

[27] N. Garcia-Hernandez, N. Tsagarakis, and D. Caldwell, "Feeling through tactile displays: A study on the effect of the array density and size on the discrimination of tactile patterns," IEEE Transactions on Haptics, vol. 4, no. 2, pp. $100-110,2011$. 
[28] K. Minamizawa, S. Fukamachi, H. Kajimoto, N. Kawakami, and S. Tachi, "Gravity grabber: wearable haptic display to present virtual mass sensation," in Proc. ACM SIGGRAPH, Emerging technologies, vol. 1, 2007, pp. 250-258.

[29] M. Wijntjes, A. Sato, V. Hayward, and A. Kappers, "Local surface orientation dominates haptic curvature discrimination," IEEE Transactions on Haptics, vol. 2, no. 2, pp. $94-102,2009$.

[30] A. Ferber, M. Peshkin, and J. Colgate, "Using kinesthetic and tactile cues to maintain exercise intensity," IEEE Transactions on Haptics, vol. 2, no. 4, pp. 224-235, 2009.

[31] K. Minamizawa, D. Prattichizzo, and S. Tachi, "Simplified design of haptic display by extending one-point kinesthetic feedback to multipoint tactile feedback," in Proc. IEEE Haptic Symposium 2010, vol. 1, 2010, pp. 257-260.

[32] D. Prattichizzo, C. Pacchierotti, S. Cenci, K. Minamizawa, and G. Rosati, "Using a fingertip tactile device to substitute kinesthetic feedback in haptic interaction," in Haptics: Generating and Perceiving Tangible Sensations. Eurohaptics 2010, ser. Lecture Notes in Computer Science, 2010, pp. 125-130.

[33] I. Birznieks, P. Jenmalm, A. W. Goodwin, and R. S. Johansson, "Encoding of direction of fingertip forces by human tactile afferents," Journal of Neuroscience, vol. 21, no. 20, pp. 8222-8237, 2001.

[34] V. Hayward, O. Astley, M. Cruz-Hernandez, D. Grant, and G. Robles-De-La-Torre, "Haptic interfaces and devices," Sensor Review, vol. 24, no. 1, pp. 16-29, 2004.

[35] F. Chinello, M. Malvezzi, C. Pacchierotti, and D. Prattichizzo, "A three DoFs wearable tactile display for exploration and manipulation of virtual objects," in Proc. IEEE Haptic Symposium 2012, 2012.

[36] B. Dasgupta and T. S. Mruthyunjaya, "The stewart platform manipulator: a review," Mechanism and machine theory, vol. 35, no. 1, pp. 15-40, 2000.

[37] L. Rosenberg, "Virtual fixtures: perceptual tools for telerobotic manipulation," in Proc. IEEE Virtual reality annual international symposium, vol. 1, 1993, pp. 76-82.

[38] J. Abbott, P. Marayong, and A. Okamura, "Haptic virtual fixtures for robot-assisted manipulation," Robotics research, vol. 28, no. 1, pp. 49-64, 2007.

[39] B. Davies, "Robotic devices in surgery," Minimally invasive therapy and allied technologies, vol. 12, no. 1, pp. 5-13, 2003.

[40] S. Cotin, H. Delingette, and N. Ayache, "Real-time elastic deformations of soft tissues for surgery simulation," IEEE Transactions on Visualization and Computer Graphics, vol. 5, no. 1, pp. 62-73, 1999.

[41] C. Zilles and J. Salisbury, "A constraint-based god-object method for haptic display," in Proc. IEEE/RSJ International Conference on Intelligent Robots and Systems, vol. 3, 1995, pp. 146-151.

[42] I. Nisky, A. Pressman, C. M. Pugh, F. A. Mussa-Ivaldi, and A. Karniel, "Perception and action in teleoperated needle insertion," IEEE Transactions on Haptics, vol. 4, no. 3, pp. 155-166, 2011.

[43] T. Lam, M. Mulder, and M. Van Paassen, "Haptic feedback in UAV tele-operation with time delay," Journal of guidance, control $\mathcal{E}$ dynamics, vol. 31, no. 6, pp. 1728-1739, 2008.

[44] E. Kandel, J. Schwartz, and T. Jessell, Principles of neural science, 4th ed. McGraw-Hill New York, 2000.

[45] G. Robles-De-La-Torre, "The importance of the sense of touch in virtual and real environments," IEEE Multimedia, vol. 13, no. 3, pp. 24-30, 2006.

[46] J. Jeka et al., "The structure of somatosensory information for human postural control," Motor Control, vol. 2, no. 1, pp. 13-33, 1998.

[47] S. Hirche, A. Bauer, and M. Buss, "Transparency of haptic telepresence systems with constant time delay," in Proc. IEEE Conference on Control Applications, vol. 1, 2005, pp. 328-333.

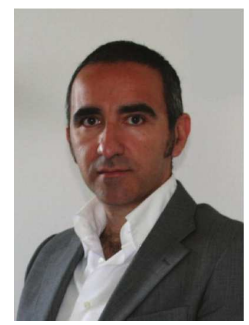

Domenico Prattichizzo (S'93 - M'95) received the M.S. degree in Electronics Engineering and the Ph.D. degree in Robotics and Automation from the University of Pisa in 1991 and 1995, respectively. Since 2002 Associate Professor of Robotics at the University of Siena. Since 2009 Scientific Consultant at Istituto Italiano di Tecnologia, Genova Italy. In 1994, Visiting Scientist at the MIT Al Lab. Co-author of the Grasping chapter of Handbook of Robotics Springer, 2008, awarded with two PROSE Awards presented by the American Association of Publishers. Since 2007 Associate Editor in Chief of the IEEE Trans. on Haptics. From 2003 to 2007, Associate Editor of the IEEE Trans on Robotics and IEEE Trans. on Control Systems Technologies. Vice-chair for Special Issues of the IEEE Technical Committee on Haptics (2006-2010). Chair of the Italian Chapter of the IEEE RAS (2006-2010), awarded with the IEEE 2009 Chapter of the Year Award. Co-editor of two books by STAR, Springer Tracks in Advanced Robotics, Springer $(2003,2005)$. Research interests are in haptics, grasping, visual servoing, mobile robotics and geometric control. Author of more than 200 papers in these fields.

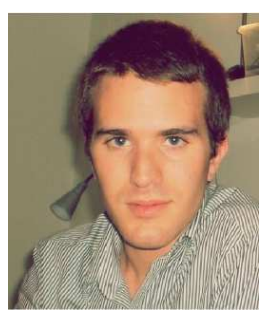

Claudio Pacchierotti (S'12) received the M.S. degree con lode in computer engineering in 2011 from the University of Siena, Italy. He was an exchange student at the Karlstad University, Sweden in 2010. He is currently a Ph.D. student at the Department of Information Engineering of the University of Siena and at the Department of Advanced Robotics of the Italian Institute of Technology. His research interests include robotics and haptics, focusing on cutaneous force feedback techniques and humanrobot interfaces for medical applications.

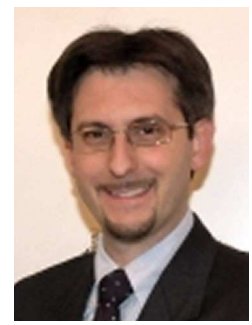

Giulio Rosati received the M.S. degree with honors in Mechanical Engineering from the University of Padua, Italy (1999) and the Ph.D. degree in Machine Mechanics from the University of Brescia, Italy (2003). He is Associate Professor (since 2006) and was Assistant Professor (2004-2006) of Machine Mechanics at the University of Padua, Italy. He was Visiting Professor at the University of California at Irvine in 2007. His research interests include haptics, robotics, industrial automation and medical and rehabilitation robotics. Dr. Rosati is member of the Faculty Board of the Doctoral School in Mechatronics Engineering of the University of Padua, Italy (since 2007). 